

\title{
Mesures précises à l'aide d'un détecteur courbe
}

\author{
J. Roux, M. Volfinger
}

\section{To cite this version:}

J. Roux, M. Volfinger. Mesures précises à l'aide d'un détecteur courbe. Journal de Physique IV Proceedings, 1996, 06 (C4), pp.C4-127-C4-134. 10.1051/jp4:1996413 . jpa-00254296

\section{HAL Id: jpa-00254296 https://hal.science/jpa-00254296}

Submitted on 1 Jan 1996

HAL is a multi-disciplinary open access archive for the deposit and dissemination of scientific research documents, whether they are published or not. The documents may come from teaching and research institutions in France or abroad, or from public or private research centers.
L'archive ouverte pluridisciplinaire HAL, est destinée au dépôt et à la diffusion de documents scientifiques de niveau recherche, publiés ou non, émanant des établissements d'enseignement et de recherche français ou étrangers, des laboratoires publics ou privés. 


\title{
Mesures précises à l'aide d'un détecteur courbe
}

\author{
J. Roux et M. Volfinger
}

CNRS-CRSCM, 1 A rue de la Férollerie, 45071 Orléans cedex 2, France

Résumé : L'utilisation d'un détecteur courbe en diffraction des rayons $\mathrm{X}$ offre de nombreux avantages, mais il faut utiliser un protocole de calibration et de mesure particulier pour obtenir des résultats précis. Pour évaluer la résolution et la reproductibilité des mesures, et pour caractériser la nature et l'importance des corrections à apporter à une calibration linéaire, on a réalisé sur une longue période des diffractogrammes d'un même mélange standard.

Abstract: Position sensitive curved detectors offers various advantages in XRD experiments. However a specific calibration and measurement protocol is required in order to achieve accurate results. To estimate the resolution and reproducibility of the measurements, and to assess the shape and size of the systematic deviations from the best linear calibration of the detector setup, diffractograms were obtained on a standard mixture over a long period of time.

\section{Introduction}

L'utilisation et le développement depuis quelques années des détecteurs courbes à localisation se sont inscrits dans une nouvelle approche à la diffraction des rayons X [1]. Ceux-ci apportent en effet de nombreux avantages, au prix de la mise en ouvre d'un protocole de calibration assez élaboré, tel qu'il a été décrit par Evain et al. [4].

Nous rapportons ici des expériences réalisées pour la mise en service d'un tel détecteur courbe (INEL CPS120) au CRSCM (Orléans). On a réalisé sur une longue durée un contrôle systématique de cet appareil en réalisant les diffractogrammes d'un mélange de standards produisant 38 pics de diffraction entre 24 et $120^{\circ}$, dans le but de tester la résolution, la stabilité et la nature des corrections de linéarisation.

\section{Méthode expérimentale}

On travaille avec une configuration de type Debye-Scherrer. L'échantillon de quelques mg est situé dans un capillaire de verre à parois de $10 \mu \mathrm{m}$ et d'absorption linéaire de $7 \times 10^{-3} \mu m^{-1}$. Il est centré dans le cercle de focalisation à l'aide d'une tête goniométrique tournante. Le détecteur de l'installation est un INEL CPS120 de rayon de cour- 
TAB. 1 - Résultats des ajustements de pics sur le diffractogramme présenté dans la figure 2 ( $^{*}=$ paramètre fixé).

Results of the peak fitting calculations for diffractogram shown in figure $2\left(^{*}=\right.$ constrained parameter\}.

\begin{tabular}{|c|c|c|c|c|c|c|c|c|c|}
\hline std & $\mathrm{hkl}$ & $2 \theta$ & $2 \theta($ lin. $)$ & $\sigma_{2 \theta}$ & $\Delta 2 \theta$ & FWHM & $\sigma_{\text {FWHM }}$ & I & $\mathrm{G} / \mathrm{L}$ \\
\hline $\mathrm{qz}$ & 100 & 24.259 & 24.292 & 0.0004 & 0.033 & 0.196 & 0.0012 & 11237 & 0.8 \\
\hline$q z$ & 101 & 31.049 & 31.007 & 0.0001 & -0.042 & 0.184 & 0.0004 & 64865 & 0.8 \\
\hline si & 111 & 33.151 & 33.132 & 0.0004 & -0.019 & 0.176 & 0.0012 & 9973 & 0.8 \\
\hline$q z$ & 110 & 42.699 & 42.732 & 0.0006 & 0.033 & 0.180 & 0.0018 & 5182 & 0.7 \\
\hline$q z$ & 102 & 46.155 & 46.189 & 0.0006 & 0.034 & 0.174 & 0.0016 & 5186 & 0.8 \\
\hline$q z$ & 111 & 47.139 & 47.166 & 0.0010 & 0.027 & 0.178 & 0.0028 & 2387 & 0.8 \\
\hline$q z$ & 200 & 49.737 & 49.745 & 0.0007 & 0.008 & 0.180 & 0.0022 & 3716 & 0.7 \\
\hline$q z$ & 201 & 53.737 & 53.724 & 0.0009 & -0.013 & 0.182 & 0.0026 & 2570 & 0.8 \\
\hline si & 220 & 55.531 & 55.553 & 0.0005 & 0.022 & 0.174 & 0.0014 & 6895 & 0.7 \\
\hline$q z$ & 112 & 58.950 & 58.923 & 0.0004 & -0.027 & 0.180 & 0.0012 & 10236 & 0.7 \\
\hline$q z$ & 003 & 59.519 & 59.486 & 0.0072 & -0.033 & 0.196 & 0.0148 & 209 & $0.7^{*}$ \\
\hline $\mathrm{qz}$ & 202 & 64.689 & 64.635 & 0.0009 & -0.054 & 0.196 & 0.0026 & 2810 & 0.7 \\
\hline $\mathrm{qz}$ & 103 & 65.250 & 65.175 & 0.0017 & -0.075 & 0.192 & 0.0050 & 1245 & 0.7 \\
\hline si & 311 & 66.220 & 66.178 & 0.0007 & -0.042 & 0.188 & 0.0020 & 4007 & 0.7 \\
\hline $\mathrm{qz}$ & 210 & 67.587 & 67.498 & 0.0080 & -0.089 & 0.200 & 0.0166 & 169 & $0.7^{*}$ \\
\hline $\mathrm{qz}$ & 211 & 70.923 & 70.839 & 0.0006 & -0.084 & 0.212 & 0.0016 & 6558 & 0.6 \\
\hline $\mathrm{q} z$ & 113 & 75.956 & 75.901 & 0.0018 & -0.055 & 0.216 & 0.0056 & 1167 & 0.6 \\
\hline $\mathrm{qz}$ & 300 & 78.160 & 78.101 & 0.0076 & -0.059 & 0.300 & $*$ & 213 & $0.7^{*}$ \\
\hline $\mathrm{qz}$ & 212 & 80.668 & 80.593 & 0.0014 & -0.075 & 0.264 & 0.0038 & 3212 & $0.7^{*}$ \\
\hline $\mathrm{qz}$ & 203 & 81.150 & 81.109 & 0.0017 & -0.041 & 0.300 & $*$ & 3893 & $0.7^{*}$ \\
\hline $\mathrm{q} z$ & 301 & 81.393 & 81.348 & 0.0040 & -0.045 & 0.300 & $*$ & 1710 & $0.7^{*}$ \\
\hline si & 400 & 82.420 & 82.389 & 0.0027 & -0.031 & 0.262 & 0.0084 & 747 & 0.9 \\
\hline $\mathrm{qz}$ & 104 & 87.971 & 87.937 & 0.0018 & -0.034 & 0.278 & 0.0052 & 1317 & 0.7 \\
\hline $\mathrm{qz}$ & 302 & 90.842 & 90.801 & 0.0015 & -0.041 & 0.282 & 0.0046 & 1782 & 0.6 \\
\hline si & 331 & 91.770 & 91.778 & 0.0018 & 0.008 & 0.282 & 0.0054 & 1398 & 0.6 \\
\hline $\mathrm{qz}$ & 220 & 93.457 & 93.437 & 0.0025 & -0.020 & 0.300 & 0.0070 & 916 & 0.8 \\
\hline$q z$ & 114 & 98.101 & 98.038 & 0.0023 & -0.063 & 0.306 & 0.0068 & 1639 & 0.3 \\
\hline$q z$ & 310 & 98.538 & 98.493 & 0.0023 & -0.045 & 0.330 & 0.0060 & 1513 & 0.8 \\
\hline $\mathrm{qz}$ & 311 & 101.729 & 101.693 & 0.0026 & -0.035 & 0.338 & 0.0084 & 1001 & 0.5 \\
\hline $\mathrm{qz}$ & 303 & 106.784 & 106.920 & 0.0045 & 0.136 & 0.356 & 0.0132 & 538 & 0.7 \\
\hline si & 422 & 107.581 & 107.652 & 0.0016 & 0.071 & 0.348 & 0.0050 & 1929 & 0.6 \\
\hline $\mathrm{qz}$ & 312 & 111.631 & 111.631 & 0.0018 & 0.000 & 0.394 & 0.0052 & 1721 & 0.7 \\
\hline $\mathrm{qz}$ & 400 & 114.508 & 114.553 & 0.0066 & 0.045 & 0.400 & $*$ & 345 & $0.7^{*}$ \\
\hline$q z$ & 105 & 117.264 & 117.298 & 0.0038 & 0.034 & 0.400 & $*$ & 951 & $0.7^{*}$ \\
\hline si & 511 & 117.697 & 117.927 & 0.0028 & 0.230 & 0.542 & 0.0078 & 1442 & 0.9 \\
\hline$q z$ & 214 & 119.648 & 119.770 & 0.0029 & 0.122 & 0.448 & 0.0088 & 982 & 0.7 \\
\hline
\end{tabular}




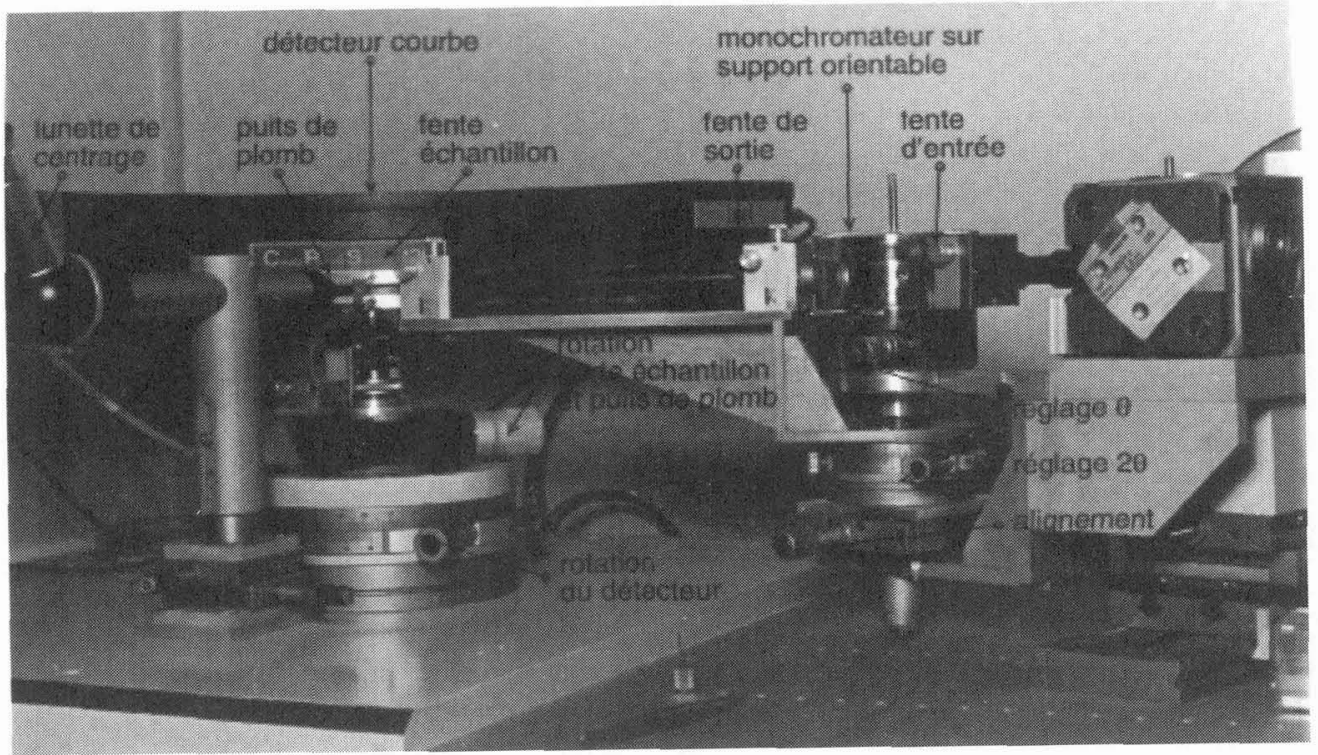

FIG. 1 - Vue générale du montage diffractométrique $\mathrm{X}$ à poudre avec le détecteur courbe. Powder X-ray diffractommetry setup using a position sensitive curved detector.

bure $250 \mathrm{~mm}$, ouvrant sur $120^{\circ}$. Un flux gazeux à faible débit d'un mélange $85 \%$ argon $15 \%$ éthane y circule sous une pression de 6.5 bar. La haute tension positive de $9650 \mathrm{~V}$ appliquée à l'anode correspond au plateau du détecteur à gaz.

L'installation du détecteur, du monochromateur et du puits ont été modifiées comme il est indiqué sur la figure 1 . Le faisceau de RX est focalisé par réflexion sur les plans 101 d'un cristal de quartz courbé sur un rayon de $1400 \mathrm{~mm}$ et taillé à $8^{\circ}$ (type Johann). $\mathrm{Ce}$ monochromateur est monté solidement sur un petit goniomètre permettant un réglage fin des angles $\theta$ et $2 \theta$ de diffraction des raies issues de l'anode. Le support des fentes de collimation du faisceau est solidaire du support du monochromateur (figure 1). Le faisceau direct est arrêté par un puits de $\mathrm{Pb}$ disposé dans une glissière solidaire du bâti, permettant de limiter le parcours du faisceau direct à l'air libre au strict minimum: $30 \mathrm{~mm}$.

Le tube de RX à anode de Co est soumis à une haute tension de $30 \mathrm{kV}$, et le débit électronique utilisé habituellement est compris entre 20 et $30 \mathrm{~mA}$. La fente de sortie vers le capillaire a été fixée en permanence à une largeur de $250 \mu \mathrm{m}$. La position $0^{\circ}$ est marquée dans le détecteur à l'aide du faisceau direct atténué par un absorbeur d'aluminium épais qui ne laisse passer qu'un taux de comptage semblable à celui des raies de diffraction. Pour toutes les mesures présentées ici, les réglages de l'électronique associée ont été fixés et les conditions sont donc restées les mêmes dans les limites de stabilité des composants. La baie reste constamment alimentée, jour et nuit, pour optimiser la stabilité de 
l'électronique. Les spectres sont acquis dans une mémoire tampon autonome. Le champ angulaire du détecteur courbe est distribué dans 4096 canaux, correspondant à un pas de $0.0293^{\circ} 2 \theta /$ canal avec un gain de conversion du codeur sélectionné à $1 \mathrm{mV} /$ canal.

Les standards utilisés sont (a) du quartz $\alpha$ de synthèse de qualité optique, monocristallin broyé $(\leq 5 \mu m)$ et (b) du silicium standard NBS-640a. Un mélange dans le rapport $\mathrm{Qz} / \mathrm{Si}=4 / 1$ de ces deux standards a été mis dans un capillaire en verre de $0.3 \mathrm{~mm}$ de diamètre soumis à une rotation de $1 \mathrm{t} / \mathrm{s}$. Les diagrammes ont été obtenus sur des durées variant de 30 à 200 min pendant une période s'étalant sur un mois, puis sur deux ans. Les mesures sont effectuées sur les 36 raies données dans le tableau 1 .

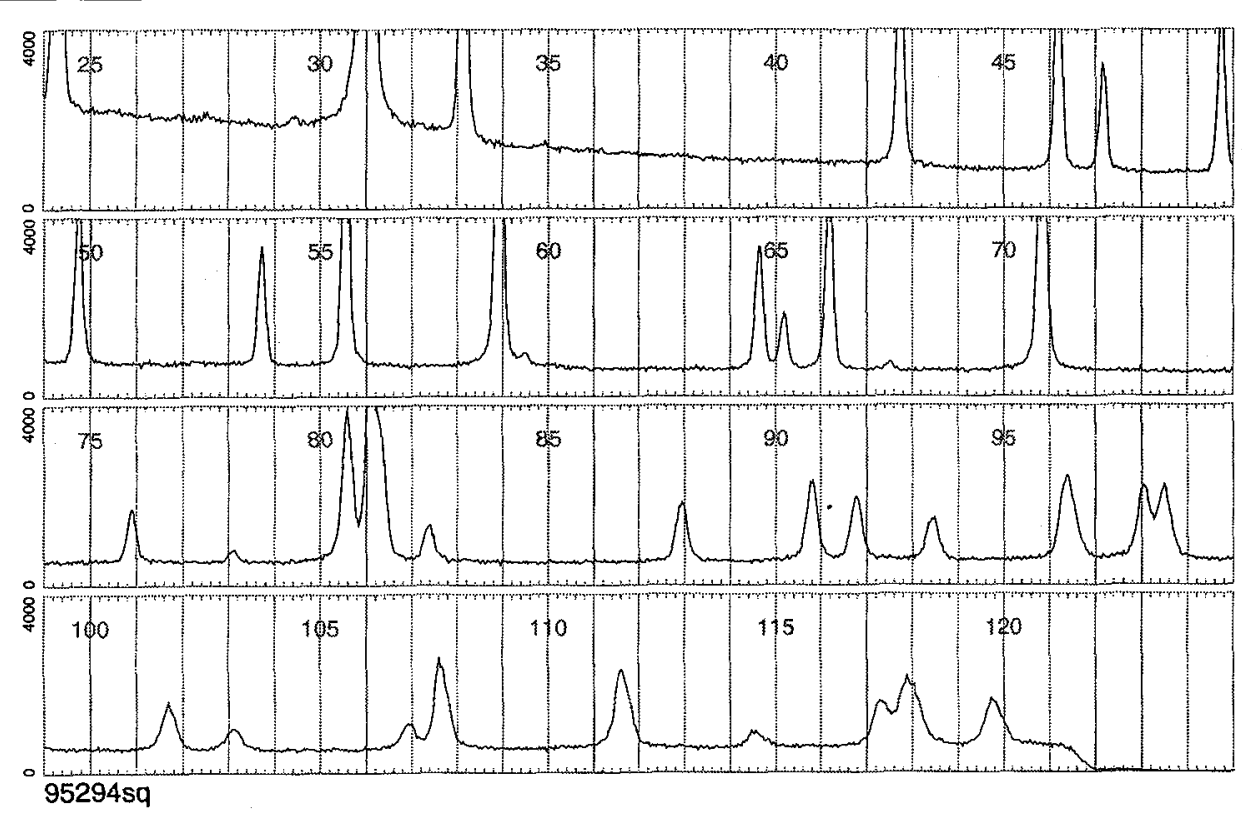

FIG. 2 - Diffractogramme obtenu pour le mélange de standards silicium et quartz. (CoK $\alpha_{1}$ focalisée, $30 \mathrm{kV}, 20 \mathrm{~mA}$, capillaire $\varnothing=0.3 \mathrm{~mm}$, géométrie $\mathrm{DS}$, fente $250 \mu \mathrm{m}$, acquisition : 800 $\min$ ).

Diffractogram obtained with a standard mixture of quartz and silicon. (CoK $\alpha_{1}$ focalised, $30 \mathrm{~V}, 20 \mathrm{~mA}$, glass capillary $\emptyset=0.3 \mathrm{~mm}$, DS setup, slit width $250 \mu \mathrm{m}$, acquisition time: $800 \mathrm{~min}$ ).

\section{Traitement informatique des mesures}

Chaque spectre (cf. Fig. 2), est transféré sur un ordinateur et analysé au moyen de programmes informatiques de traitement et d'édition de données spectroscopiques [3] mis au point au CRSCM. On a mesuré systématiquement tous les paramètres des pics de diffraction de chaque diagramme, comme décrit ci-après. 
Pour chaque type de standard les données suivantes sont préparées: (1) les zones angulaires, de part et d'autre du ou des pics à mesurer, où le bruit de fond peut être mesuré, (2) une estimation $2 \theta$ de la position des pics de diffraction à ajuster, (3) de leurs largeur à mi-hauteur FWHM, (4) des coefficients de pondération G/L entre pic de forme gaussienne ou lorentzienne.

Les différents paramètres peuvent être ajustés ou fixés. Dans le cas de pics multiples, on peut en particulier imposer FWHM et la forme du pic à partir de mesures précédentes pour améliorer la stabilité du calcul. Les mesures sont pondérées en $1 / \sigma^{2}$ avec $\sigma=\sqrt{n}$. Les résultats des ajustements (y compris les matrices de variance/covariance) sont fournis dans un fichier ASCII. Des petits programmes de post-traitement permettent de mettre en forme les résultats. La figure 3 donne deux exemples de ces calculs présentés sous forme graphique. Le logiciel SPC d'édition et de calcul de diffractogrammes fonctionne de façon intéractive (graphique) ou en traitement par lot, ce qui permet l'automatisation des opérations. Le traitement complet d'un diffractogramme y compris les post-traitements graphiques est effectué en moins de 10 secondes (PC486/66). Les sorties graphiques sont sous forme de fichiers PostScript.

Pour chaque mesure on établit une calibration linéaire en utilisant des pics isolés de forte intensité (e.g. Qz100, Qz110, Qz112, Qz211, Qz104, Si422, Qz312). Pour ceux ci, la précision de l'initialisation du calcul n'est pas critique et le traitement automatique ne pose pas de difficulté. La calibration est obtenue par une régression des mesures par rapport aux valeurs théoriques de $2 \theta$ des fiches JCPDS 33-1161 du quartz $\alpha$ et 27-1402 du silicium.

Dans une seconde étape cette calibration établie, on mesure tous les autres pics du diagramme y compris les pics composites, avec une initialisation suffisamment précise pour n'avoir pas de problèmes de convergence.

\section{Résultats}

\subsection{Résolution angulaire}

La variation progressive de la largeur à mi-hauteur (FWHM) des divers pics de réflexion du quartz et du silicium est représentée sur la figure 4 en fonction des angles théoriques. Ces résultats sont analogues à ceux présentés par Evain et al. [4], la résolution étant toutefois moins bonne, du fait de l'utilisation d'une anode de cobalt.

\subsection{Calibration}

Les coefficients de la calibration linéaire mesurés dans l'espace d'un mois sur 7 diffractogrammes de qualité courante (acquisition entre 20 et 60 minutes) varient peu. On obtient les moyennes suivantes pour ces coefficients: ordonnée à l'origine $2 \theta=-5,170^{\circ}$ avec $1 \sigma=0,020 ;$ pas $=0,031766^{\circ} / \mathrm{canal}$ avec $1 \sigma=0,000017$. D'autres diffractogrammes de meilleure qualité (durée de 180 à $800 \mathrm{~min}$ ) ont été réalisés, dont certain récemment après réalignement $\mathrm{du}$ diffractomètre ayant entrainé un décalage d'environ 0,9 degré dans la position du détecteur par rapport au faisceau direct. La pente moyenne sur un an ne présente pas de dérive importante: 0.031726 avec $1 \sigma=0,000030$. Ceci indique la bonne stabilité de la chaîne de mesure. 



FIG. 3 - Résultats d'ajustements par moindres carrés sur un et sur plusieurs pics. Le poids statistique de chaque canal est pris égal à $1 / n, n$ : nombre de coups ( $\mathrm{F}=$ nombre de degrés de liberté à comparer à $\chi^{2}$ ).

Results of least squares fits on a single and multiple peaks. The statistical wheight of each channel is taken equal to $1 / n, n$ is the number of counts ( $F$ is the degree of freedom to compare with $\chi^{2}$ ).

Pour ces diffractogrammes, on a reporté pour tous les pics mesurables, les écarts observés entre la valeur de $2 \theta$ calculée à l'aide de la droite de calibration et les valeurs théoriques. Ces calculs sont présentés sur la figure 5 . On a distingué deux groupes de mesures effectuées à presque deux ans d'intervalle sur des diffractogrammes de qualité convenable (temps de mesure supérieur à 1 heure).

On voit que les écarts, atteignant localement $0.1^{\circ} 2 \theta$, sont d'amplitude comparable à ceux mesurés par Shishiguchi et al. [2] et Evain et al. [4].

Mais le fait primordial qui caractérise le détecteur étudié est le caractère brutal des variations des corrections de linéarisation dans des domaines angulaires étroits, variations beaucoup plus irrégulières que celles déterminées par Evain et al. (1993) [4]. Toutefois il faut souligner la remarquable stabilité dans le temps de ces écarts. Dans chaque groupe la reproductibilité des mesures est dans le domaine des barres d'erreur $( \pm 2 \sigma)$ déterminées à partir des matrices de variance/covariance des ajustements. Pour les pics de bonne statistique, situés avant $80^{\circ} 2 \theta$, les dents de scie sont reproductibles à mieux que $0,01^{\circ}$. La stabilité à long terme que l'on peut estimer en comparant les deux groupes de mesures est aussi manifeste, les petites différences observées sont dues au faible mais significatif changement de position du détecteur $\left(0.9^{\circ}\right)$ entre les deux séries de mesures.

Ces résultats montrent que l'échantillonage de l'espace angulaire réalisé avec le mélange quartz-silicium est insuffisant pour obtenir une image fidèles des écarts de linéarité. Il 


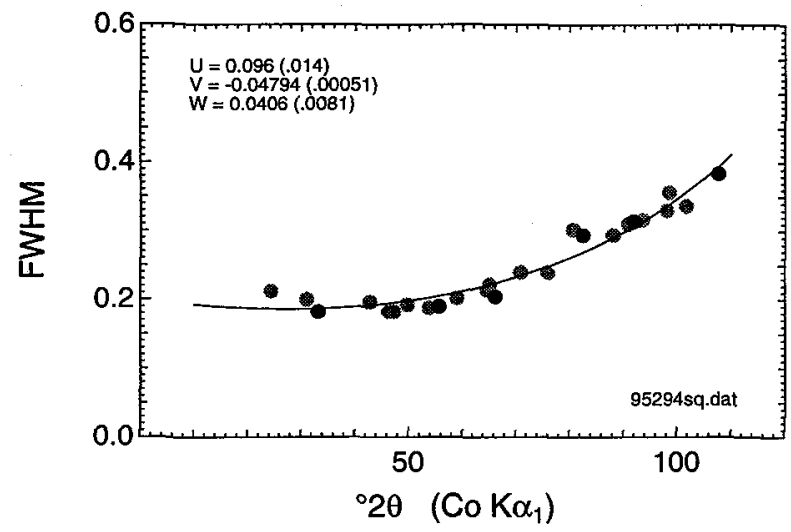

FIg. 4 - Résolution en fonction de l'angle de diffraction mesuré sur les pics du mélange de silicium (points noir) et de quartz (points gris). Les coefficients de l'équation de Caglioti[5] $\left(\sqrt{U \tan ^{2} \theta+V \tan \theta+W}\right)$ sont donnés sur la figure (erreurs=1 $\left.\sigma\right)$.

FWHM against diffraction angle for the standard silicon (black symbol) + quartz (gray symbol) mixture. Coefficients of the Caglioti[5] empirical equation $\left(\sqrt{U \tan ^{2} \theta+V \tan \theta+W}\right)$ are given on the figure (errors as $\left.1 \sigma\right)$.

semble, pour le détecteur utilisé, qu'une résolution angulaire des points de calibration de l'ordre de 1 à $2^{\circ} 2 \theta$ soit nécessaire. Deux méthodes peuvent être combinées pour obtenir ce résultat: (1) utilisation d'un standard tel que celui préconisé par Evain et al. [4], (2) réalisation d'une série de pauses en décalant progressivement le détecteur par pas d'environ $1^{\circ}$ pour combler les lacunes entre les différents pics mesurables du standard, et en repérant la position du faisceau direct.

\section{Conclusion}

Le résultat principal obtenu concerne la mise en évidence d'une courbe de calibration beaucoup plus irrégulière que celles précédemment observées pour des détecteurs de ce type. Elle est toutefois très reproductible. Cette courbe de calibration spécifique du détecteur doit être mesurée à l'aide d'un échantillonnage extrêmement serré de l'espace angulaire. Ceci est rendu possible par la bonne stabilité dans le temps de la chaîne de mesure. La procédure de calibration relativement élaborée est toutefois réalisable en 1 ou 2 jours à l'aide (1) du montage goniométrique du détecteur qui permet de modifier son orientation par rapport à celle du faisceau direct, $(2)$ des logiciels de traitement de données adaptés. Des mesures à mieux que $0.01^{\circ}$ peuvent alors être effectuées, en utilisant conventionnellement un étalon interne, pour ajuster finement les coefficients de la calibration linéaire et en appliquant les corrections spécifiques précédemment établies. Une table de correction canal par canal nous semble être la méthode la plus simple d'implémentation. 


\section{Références}

[1] Caglioti G., Paoletti A. and Ricci F. P., Nucl. Instrum. 3 (1958) 223-228.

[2] Ballon J., Comparat V. and Pouxe J., Nucl. Instrum. Methods 217 (1983) 213-216.

[3] Shishiguchi S., Minato I. and Hashizume H., J. Appl. Cryst. 19 (1986) 420-426.

[4] Roux J. et Beny C., Symposium Georaman 89 ANRT et SFMC, Toulouse (1989).

[5] Evain M., Deniard P., Jouanneaux A. and Brec R., J. Appl. Cryst. 26 (1993) 563-569.
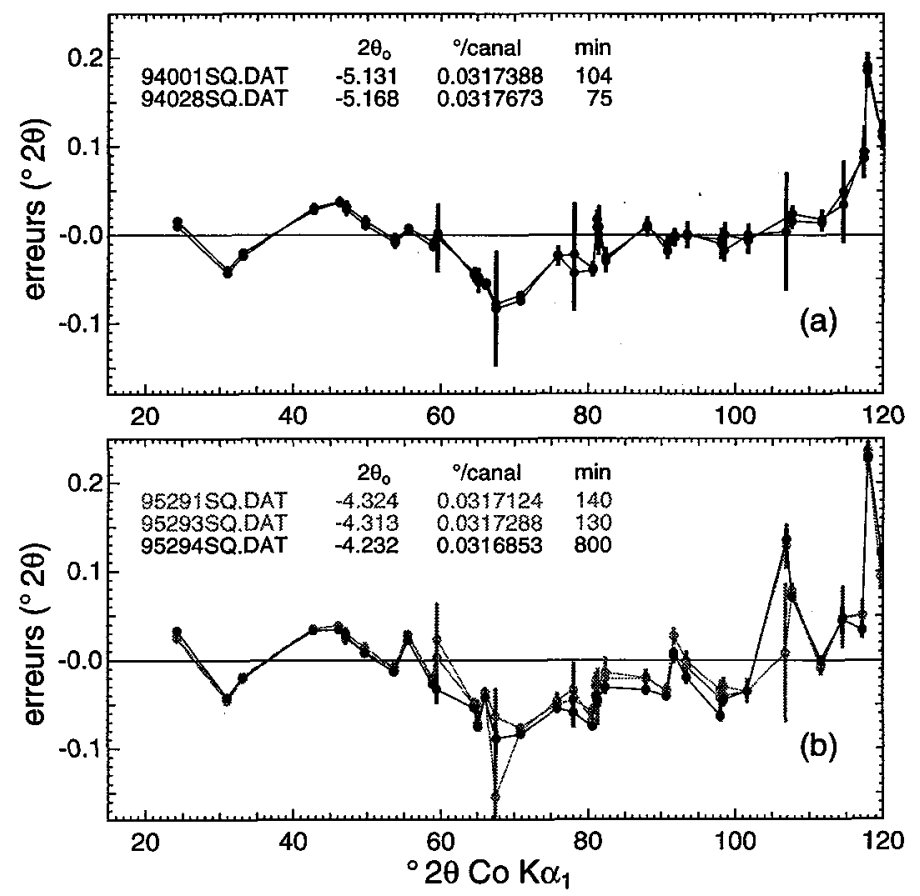

FrG. 5 - Écarts entre les valeurs angulaires théoriques et les valeurs calculées avec les calibrations linéaires dont les coefficients sont reportées sur la figure ainsi que les durées d'acquisition. Deux groupes de mesures ( $a$ et b) à environ deux ans d'intervalle y sont distingués. Les barres d'erreurs $( \pm 2 \sigma)$ dépendent de l'intensité du pic et la résolution du détecteur.

Deviations from the linear calibration whose coefficients are given on the figure along with the acquisition time. Two groups ( $a$ and $b$ ) of measurements, taken approximately two years apart are shown separately. Error bars $( \pm 2 \sigma)$ vary with the peak height and the detector resolution. 